\title{
How do we ensure safety for patients and clinicians while utilising Telemedicine in the field of Diabetes
}

\author{
Geeta Jeevan Aurangabadkar* \\ Consultant Endocrinologist, Care hospital and Excell hospital, Hyderabad, India
}

*Address for Correspondence: Dr Geeta Aurangabadkar, Excell hospital, Hyderabad, India, Email: vrgeeta@yahoo.com

Received: 25 August 2020; Accepted: 07 September 2020; Published: 08 September 2020

Citation of this article: Aurangabadkar G (2020) How do we ensure safety for patients and clinicians while utilising Telemedicine in the field of Diabetes. Rea Int J of End and Diabe. 1(1): 022-025. DOI: 10.37179/rijed.000006.

Copyright: (C) 2020 Aurangabadkar G. This is an open access article distributed under the Creative Commons Attribution License, which permits unrestricted use, distribution, and reproduction in any medium, provided the original work is properly cited.

\begin{abstract}
Telemedicine use has been at its highest for patients in recent times due to ongoing COVID 19 pandemics. Although avoidance of face to face consultations carries many advantages for both patients and clinicians, there are always concerns around safety and legal aspects. So, how do we assure that the way of using teleconsultation in the field of diabetes is a mutually safe, effective, and sustainable option in the long term? This article lays out some important solutions addressing underlying apprehension and barriers in the use of telemedicine for diabetes. This is obviously truly relevant in the current scenario of COVID 19 where we anticipate continued use of telemedicine in near future.
\end{abstract}

Keywords: COVID-19 (Coronavirus disease of 2019), Information technology (IT), Telemedicine in diabetes, Patient, and clinician safety.

\section{Introduction}

Telemedicine is a term coined in the 1970 s, which literally means "healing at a distance" [1]. "The delivery of health care services, where distance is a critical factor, by all health care professionals using information and communication technologies for the exchange of valid information for diagnosis, treatment and prevention of disease and injuries, research and evaluation, and for the continuing education of health care providers, all in the interests of advancing the health of individuals and their communities" [2].

The very purpose of telemedicine is to provide distant clinical services by overcoming geographical barriers with the help of technology with the aim to improve clinical outcomes. In Indian setting, this has been especially useful for the underprivileged areas such as certain remote areas in India since COVID-19 outbreak [9]. The usefulness of telemedicine in general has been applied to a variety of different medical and surgical branches $[10,11]$.

\section{Doctor-Patient Partnership in Diabetes Management}

Diabetes most often is a chronic progressive disease, requiring a long term ongoing follow up and treatment. For an effective outcome, patients need to demonstrate a good consistent compliance with their medications. However, the ability to comply with prescribed regime can be influenced greatly by fluctuations in their blood glucose levels, fear of hypoglycemia leading to suboptimal glycemic control. A study by Chan et al., observed that only $3.6 \%$ of Type 2 diabetes patients attain all three recommended targets [3].

Factors such as instability in blood sugars, side effects from medications, frequently changing dosage of medications and overall complexity of management of diabetes warrant more frequent contacts with the clinician concerned. For successful diabetes management, clinicians must invest required time into counselling, support the patient led self-monitoring, encourage patient empowerment, and set realistic individualized targets considering personal psychosocial 
factors. Hence, the partnership of both clinicians and patients plays a pivotal role in optimal management of diabetes in the long run.

\section{Role of Telemedicine in Diabetes}

Information technology has a great potential to address some of the challenges faced by both developed and developing countries in providing accessible, cost- effective, high-quality health care services. Rural communities in India have traditionally been deprived of access to quality health care and have suffered from lack of access to basic care. It certainly breaks the geographical barriers and bridges that gap of inequality in delivery of services by acting as a link with specialists, referral hospitals, and tertiary care centers [4].

Teleconsultations by experienced endocrinologists, timely advice on blood sugar readings and blood results, appropriate medication prescribing and insulin counselling along with active screening and monitoring for macrovascular and microvascular complications can all have a positive impact on overall health of the community. Data from meter, pump, and sensor downloads are critical to management where telemedicine offers an ideal way to interact with patients. Patient's vital signs, especially blood pressure and weight can be selfmonitored at home [5-6]. With easy accessibility of medical care, telemedicine can enable patients to seek treatment earlier and adhere better to their prescribed treatments [7] and improve the quality of life for patients with chronic conditions [8].

COVID-19 era is likely to continue for the coming months in which case, face to face consultations and examinations continue to pose a high risk of transmission of COVID 19 to both clinicians and patients. Moreover, it is ideal for diabetes, as patients are not always required to be examined unlike other specialties of medicine. Besides, diabetes patients are often frail, elderly with mobility issues and in most cases, there is no compelling need to physically see a doctor for example for blood report review. Patients who are immunocompromised benefit by shielding from communicable diseases in clinic spaces. Hence, the option of Teleconsultations in the field of diabetes for clinicians and patients seems mutually convenient, flexible, cost-effective, and practical. It also saves time and transportation costs for our diabetic patients who live in remote areas and are already overburdened with medical costs. The evidence around cost effectiveness is supported by several trials.

A 2019 meta-analysis published in the journal Telemedicine and e-Health, researchers from several university hospitals in France analyzed 42 randomized controlled trials investigating the use of telemedicine (8 studies used teleconsultation and 34 used devicebased telemonitoring) vs usual care in diabetes management [12]. They examined data from a total of 6170 patients (mean ages, 13.371.0 years) from 12 studies focused on type 1 diabetes, 21 focused on type 2 diabetes, and 9 that included both patients with type 1 and type 2 diabetes.

The results demonstrated a significantly greater mean reduction in hemoglobin $\mathrm{Alc}(\mathrm{HbAlc})$ in the telemedicine groups compared with usual care $(\mathrm{P}<.001)$, especially in trials lasting $>6$ months and in patients with type 2 diabetes compared with type 1 diabetes $(\mathrm{P}<0001)$. In addition, greater benefits were observed in older patients (aged 41-50 years or $>50$ years) compared with younger patients [12]. These observations align with findings from a 2018 meta-analysis of 19 studies that included data from a total of 6294 individuals, which showed better glycemic control with telehealth vs usual care (weighted mean difference in glycemic index, $-0.22 \%$; 95\% CI, -0.28 to -0.15 ; $\mathrm{P}<.001)$ [13].

\section{Devices for Telemedicine}

Data safety and confidentiality of patients are the cornerstone of successful telemedicine. Apart from basic telecommunications technology, like telephones, internet, and computers, access to various other devices and systems can help make telemedicine an efficient tool to use.

- 'Telemedicine Carts' are used at hospitals or other large health care systems for more complex telemedicine solutions.

- 'Telemedicine kiosks' are often expensive and usually only purchased by large companies or retail clinics to extend healthcare offerings to employees and pharmacy customers.

- Access to high resolution integrated 'digital cameras' allow healthcare professionals to take detailed medical images (for example, diabetic skin diseases) and then share them with a specialist at another location. They can be expensive and often require use by a trained medical professional.

- 'Telemedicine Kit' can help provide portable, durable frontline care in remote areas.

- 'Telemedicine software' can offer an integrated solution to multiple purposes including live video conferencing, storeand-forward telemedicine, and collecting and monitoring patient medical data remotely. This requires purchase of a compatible software system that needs to be installed on a medical practice's computers.

- 'Mobile medical devices' are often used by either healthcare professionals or patients to capture medical data and send it to a medical professional at another location, for example ECG or vital signs monitor record.

\section{Concerns/Barriers}

Even though low-cost telemedicine applications have proven to be feasible, clinically useful, sustainable, in underserved communities, its utilization has not been on a significant scale due to a variety of barriers [14].

\section{Patient Factors}

Patients may perceive lesser satisfaction levels from teleconsultation due to lack of doctor's traditional touch. Many of them may not see teleconsultation as 'actual or real' consultation. Moreover, there is always this small possibility of abuse of medications including insulin by patients or their contacts or selling medicines for money.

\section{Clinician's Factors}

From a clinician's point of view, there is always a worry about missing out the "red flags" or systemic instability in patients. In case the patients' needs to be admitted to secondary care, there may not be suitable nearby centers. In other cases, keeping a track of diabetes related complications and screening, especially foot checks are not ideally possible. Moreover, lack of clarity and uniformity on the legal aspects around documentation or method of prescribing in teleconsultations makes this process often confusing. Furthermore, 
issues with clinician's fee payment in government sectors makes telemedicine an unattractive option for many endocrinologists.

\section{Reliance on Technology}

Telemedicine is highly dependent on technology and reliable internet networks on both patient and clinician's side. Issues with unstable power supplies, availability/access of smart phones or devices in deprived regions impact greatest on telemedicine success. Moreover, it may not be an appropriate way of consultation for those with visual or hearing deficits or many elderly patients who may not be tech-savvy.

\section{Cost Implications}

Developing countries are more likely to consider resource issues such as high costs, underdeveloped infrastructure, and shortage of technical expertise to be barriers to telemedicine.

\section{Privacy and Security Risks}

Data privacy and security breach in telehealth systems can adversely affect patients' and clinicians' level of trust and willingness to adopt and use the system. This can be damaging for the doctorpatient relationship and invite future legal problems.

\section{Legal and Ethical Issues}

Clinicians may be hesitant to use teleconsultations due to fear of litigation or concerns around safety and confidentiality of the service. Aspects of patient information, privacy and confidentiality are of greater importance in telemedicine implementation.

\section{What are the Solutions}

Tie up with nearby primary health clinics: This is particularly useful for getting patient's vital observations such as temperature, pulse, BP, oxygen saturation and blood glucose checked. This basic information aids the consulting clinician to rule out any major systemic illness or significant hyperglycaemia prompting admission. Foot checks for neuropathy or peripheral vascular disease can also be done by a local physician if tie ups done with suitable professionals locally.

Access to emergency care: Telehealth institutions should establish red flag or emergency protocols that prompt telehealth workers to direct patients to urgent care or emergency secondary care. All the staff involved in the teleconsultation team must be made aware of the protocols through mandatory training and mock workshops.

Recall system for checkups: Automated reminders for patients for retinopathy screening, diabetic foot checks, annual blood tests, cardiac checkups, urine ACR can be of great help to avoid patients slipping through the net. Indeed, this also plays a big role in prevention of microvascular and macrovascular complications secondary to diabetes.

Confidential computer software: It is imperative that the providers of telemedicine have a customized software which includes a 'confidential' electronic medical records system with different user interfaces for all diabetes patients. This is also mandatory for safe 'data sharing' such as lab reports, retinopathy photos and clinical records between different health professionals.

Robust technology and network: High speed internet, clear video and audio features and user-friendly technology are key parts of smooth and successful teleconsultations.

Prompt IT support: This is essential to clear any glitches in the system during consultation and help make the process smooth and time-efficient for both clinicians and patients.

MDT: An active multidisciplinary team (MDT) of physicians, nurses, dieticians, diabetes educators, pharmacists, and psychologists in place would help patients access all the required services under one roof conveniently.

Maintaining quality control: This can be done through auditing and appraising the consultants regularly and encouraging active participation of staff in safeguarding and significant event meetings. Providing such open opportunities helps maintain a good patient safety culture. from certain telemedicine cases.

Preventing abuse of the system: Safety measures in place such as strict patient ID (identity) proof check, clear prescription record and keeping a log of the amount of prescriptions ordered by patient would prevent misuse of medications by patients.

Litigation worries for the clinician: Patient ID checks and consent, thorough consultations notes or documentation including safety netting, confidentiality and data protection, back of medical insurance, adopting prescription methods as recommended by the local or national medical authorities, escalation protocols and rigorous training systems in place for staff would help alleviate some concerns about litigation.

\section{Conclusion}

Telemedicine for diabetes can help improve the quality of diabetes care by altering the caring environment and care delivery process especially in COVID-19 time. It is a great tool for self-management for diabetic patients and helps them to take control of their own disease management.

If implemented appropriately in underprivileged areas, telehealth can be an incredibly effective approach to ensuring patient and provider safety during this unprecedented outbreak. It offers a unique opportunity to implement innovative, safe, cost effective and quality patient care. Patients with mobility challenges, elderly could specifically benefit from this service, given their vulnerability and higher risk to exposure of infection in face to face clinic setting. However, clinicians need to keep in mind that face-to-face contact between patients and healthcare providers is invaluable and physical contact is a necessity in eye and foot examinations and in regular blood sampling for periodic testing. In summary, use of telemedicine in diabetes can be a powerful tool if essential measures are in place to ensure safety of patients and clinicians if used judiciously.

\section{References}

1. Strehle EM, Shabde N (2006) One hundred years of telemedicine: does this new technology have a place in paediatrics? Archives of Disease in Childhood 91: 956-959. Link: https://bit.ly/2FfhFBW

2. WHO (1998) A health telematics policy in support of WHO's HealthFor-All strategy for global health development: report of the WHO group consultation on health telematics, 11-16 December, Geneva, 1997. Geneva, World Health Organization. Link: https://bit. ly/3lWnWnc

3. Chan JC, Gagliardino JJ, Baik SH, Chantelot JM, Ferreira SR, et al. 
Citation: Aurangabadkar G (2020) How do we ensure safety for patients and clinicians while utilising Telemedicine in the field of Diabetes. Rea Int J of End and Diabe. 1(1): 022-025. DOI: 10.37179/rijed.000006.

(2009) Multifaceted determinants for achieving glycemic control: The International Diabetes Management Practice Study (IDMPS) Diabetes Care 32: 227-233. Link: https://bit.ly/2ZbdUoA

4. Heinzelmann PJ, Lugn NE, Kvedar JC (2005) Telemedicine in the future. Journal of Telemedicine and Telecare 11: 384-390. Link: https://bit.ly/2ZamX8X

5. Brandling-Bennett HA, Iris K, Daniel JP, Gary J, Graham JG, et al. (2005) Delivering health care in rural Cambodia via store-and-forward telemedicine: a pilot study. Telemedicine Journal and e-Health 11 56-62. Link: https://bit.ly/2FdxYiG

6. Pradhan MR (2004) ICTs application for better health in Nepal. Kathmandu University Medical Journal 2: 157-163. Link: https://bit ly/357xoy6

7. Kvedar J, Heinzelmann PJ, Jacques G (2006) Cancer diagnosis and telemedicine: a case study from Cambodia. Annals of Oncology 17: S37-S42. Link: https://bit.ly/3jPslXd

8. Chanussot-Deprez C, Contreras-Ruiz J (2008) Telemedicine in wound care. International Wound Journal 5: 651-654. Link: https://bit. ly/2GvhyTy

9. Mishra A (2003) Telemedicine in otolaryngology (an Indian perspective). Indian Journal of Otolaryngology and Head and Neck Surgery 55: 211-212. Link: https://bit.ly/35e17FK
10. Froelich W, Soeung S, Ngoun C, David P (2009) Case report: an example of international telemedicine success. Journal of Telemedicine and Telecare 15: 208-210. Link: https://bit.ly/322EHFc

11. vinals F, Mandujano L, Vargas G, Giuliano A (2005) Prenatal diagnosis of congenital heart disease using four-dimensional spatio-temporal image correlation (STIC) telemedicine via an internet link: a pilot study. Ultrasound in Obstetrics \& Gynecology 25: 25-31. Link: https:// bit.ly/357ys54

12. Tchero H, Kangambega P, Briatte C, Brunet-Houdard S, Retali GR, et al. (2019) Clinical effectiveness of telemedicine in diabetes mellitus: a meta-analysis of 42 randomized controlled trials. Telemed J E Health 25: 569-583. Link: https://bit.ly/2R0aKj1

13. Wu C, Wu Z, Yang L, Wenjun Z, Meng Z, et al. (2018) Evaluation of the clinical outcomes of telehealth for managing diabetes: a PRISMAcompliant meta-analysis. Medicine (Baltimore) 97: e12962. Link: https://bit.ly/3bzlv56

14. Wootton R (2008) Telemedicine support for the developing world. Journal of Telemedicine and Telecare 14: 109-114. Link: https://bit. ly/331FoOA 